\title{
Abnormal Expression of the Novel Epidermal Enzyme, Glucosylceramide Deacylase, and the Accumulation of its Enzymatic Reaction Product, Glucosylsphingosine, in the Skin of Patients with Atopic Dermatitis
}

\author{
Mutsumi Ishibashi, Junko Arikawa, Reiko Okamoto, Makoto Kawashima, \\ Yutaka Takagi, Kenji Ohguchi, and Genji Imokawa \\ Department of Dermatology (MI, JA, MK, Gl), Tokyo Women's Medical University, Tokyo, and Kao Biological \\ Science Laboratories (YT, KO, GI), Tochigi, Japan
}

\begin{abstract}
SUMMARY: To clarify mechanisms underlying acylceramide deficiency as an causative factor of the permeability barrier disruption seen in the skin of patients with atopic dermatitis (AD), we hypothesized and then demonstrated the presence of a novel epidermal enzyme, termed glucosylceramide (GC) deacylase. This enzyme hydrolyzes (acyl)GC at the N-acyl site to yield its lysoform, glucosylsphingosine (GS), instead of the formation of (acyl)ceramides by $\beta$-glucocerebrosidase. Assays of enzymatic activity using [palmitic acid- ${ }^{14} \mathrm{C}$ ] GC as a substrate revealed that extracts from the stratum corneum and from the epidermis (but not from the dermis) of patients with $A D$ have the significantly higher potential to hydrolyze $G C$ at the $\mathrm{N}$-acyl site to release ${ }^{14} \mathrm{C}$-labeled free fatty acid than of healthy controls. To determine the in vivo physiologic function of this novel enzyme, we measured the metabolic product GS in the upper stratum corneum. In both the involved and the uninvolved stratum corneum from patients with $A D$, there were significant increases in the amounts of GS compared with healthy controls and there was a significant inverse correlation with the decreased content of ceramides or ceramide-1 (acylceramide). Thus, collectively these results strongly suggest the physiologic relevance of GC deacylase to the acylceramide deficiency seen in the stratum corneum of patients with AD. (Lab Invest 2003, 83:397-408).
\end{abstract}

\begin{abstract}
A deficiency of ceramides in the stratum corneum is an causative factor of the barrier-disrupted and dry skin of patients with atopic dermatitis (AD) (Imokawa et al, 1991). This dysfunction of the stratum corneum leads to a high vulnerability to irritants or allergens, which results in the induction, recurrence, or refractory nature of the dermatitis. Thus, it is intriguing to determine the biochemical mechanism(s) underlying the ceramide deficiency because it may be linked to the physiopathogenesis of $A D$. In the epidermis of patients with $A D$, we have previously demonstrated the accentuated expression of a hitherto undiscovered epidermal enzyme, termed sphingomyelin (SM) deacylase, which was associated with the reduced ceramide mass in the stratum corneum as a result of its competition with the ceramideproducing enzyme sphingomyelinase (SMase) for the
\end{abstract}

DOI: 10.1097/01.LAB.0000059931.66821.92

Received November 7, 2002.

This study was performed as part of a research project on the Control of Atopic Dermatitis by Skin Care: Regulatory Factor of Cutaneous Permeability, and was supported by the special coordination funds from the ministry of education, culture, sports, science, and technology of the Japanese Government. Present address for Dr. Kenji Ohguchi, Gifu International Institute of Biotechnology, Mitake, Kani-gun, Gifu 505-0116, Japan. Address reprint requests to: Dr. Genji Imokawa, Kao Biological Science Laboratories, 2606 Akabane, Ichikai-machi, Haga, Tochigi 321-34 Japan.E-mail:imokawag@dream.ocn.ne.jp common substrate SM (Hara et al, 2000; Murata et al, 1996). Recently, it was reported that omega-OH ceramide, an important acylceramide precursor, does not derive from SM in the mammalian stratum corneum, which suggests that it derives solely from glucosylceramide (GC) precursors (Uchida et al, 2000). Thus, it is likely that acylceramides are biosynthesized not by the hydrolysis of SM but through pathways involving the deglucosylation of acylglucosylceramides by $\beta$-glucocerebrosidase (GICdase). On the basis of these findings, the acylceramide deficiency observed in $A D$ could not be explained in terms of the up-regulation of SM deacylase. As a resolution for this discrepancy, we hypothesized the existence of a novel epidermal enzyme, termed here GC deacylase, which hydrolyzes (acyl)GC at the N-acyl site to yield its lysoform, glucosylsphingosine (GS), instead of the formation of (acyl)ceramides by GICdase.

In this study, using [palmitic acid- ${ }^{14} \mathrm{C}$ ] GC as a substrate, we examined the activity of GC deacylase in the stratum corneum and in the epidermis of patients with $A D$ and compared that with levels found in healthy controls. As additional confirmation of the functional relevance of GC deacylase to the acylceramide deficiency, we quantified the amount (per milligram of stratum corneum) of the metabolic product GS produced by GC deacylase in the stratum corneum at the nanomolar level. Here we report that 
extracts from the stratum corneum and from the epidermis of patients with $A D$ have the potential to hydrolyze $\mathrm{GC}$ at the $\mathrm{N}$-acyl site to release ${ }^{14} \mathrm{C}$-labeled free fatty acid. There are significantly higher levels of this enzymatic property in skin with $A D$ than in healthy controls. Consistent with the up-regulation of GC deacylase in skin with $A D$, there was a significant increase in the content of GS, its enzymatic reaction product, in both the involved and the uninvolved stratum corneum from patients with $A D$ compared with healthy controls. The up-regulation of GC deacylase and its metabolic product GS in skin with $A D$ strongly suggests the physiologic relevance of GC deacylase to the acylceramide deficiency in the stratum corneum of patients with AD.

\section{Results}

\section{Distinction of GC Deacylase from GICdase}

In assays to measure the release of $\left[1-{ }^{14} \mathrm{C}\right]$ palmitic acid as a criterion for GC deacylase activity, the released palmitic acid would not necessarily represent the activity of GC deacylase if both GICdase and ceramidase (CDase) are present in extracts of the stratum corneum from patients with AD. This is because the release of radiolabeled palmitic acid could also result from a two-step reaction sequence wherein GC would be initially hydrolyzed by GICdase to yield radiolabeled palmitoylsphingosine (ceramide), which in turn could be converted by acid CDase to radiolabeled palmitic acid. Thus, to determine whether the release of radiolabeled palmitic acid reflects the direct deacylation of GC or the two-step reaction sequence of deglucosylation and subsequent deacylation, we separated GC deacylase from GICdase using cationic exchange chromatography. This was based on their different $\mathrm{pl}$ values, presumably near 4.2 versus 8.0 (Higuchi et al, 2000), respectively. The release of radiolabeled free fatty acid and ceramide was then characterized. Chromatographic analysis (Fig. 1A) revealed that whereas the unadsorbed fraction was able to release radiolabeled free fatty acid but not ceramide, the adsorbed fraction eluted by an increase in salt concentration was able to release radiolabeled ceramide but not free fatty acid. Analysis of radiolabeled ceramide or free fatty acid released by the crude extract and by unadsorbed fractions from the stratum corneum of five patients with $A D$ (Fig. 1B) demonstrated that whereas the crude extracts were able to release both radiolabeled ceramide and free fatty acid, the unadsorbed fraction was able to release radiolabeled free fatty acid to an extent similar to the crude extract but was not able to release radiolabeled ceramide. This suggests that despite that the unadsorbed fractions have been depleted of GICdase, they still contain GC deacylase. This strongly suggests that the GC deacylase activity seen in crude extracts from the stratum corneum of patients with $A D$ is mainly attributable to the direct deacylation of GC but not the two-step reaction sequence consisting of deglucosylation and subsequent deacylation.

\section{A CM-Sepharose Column Chromatography}

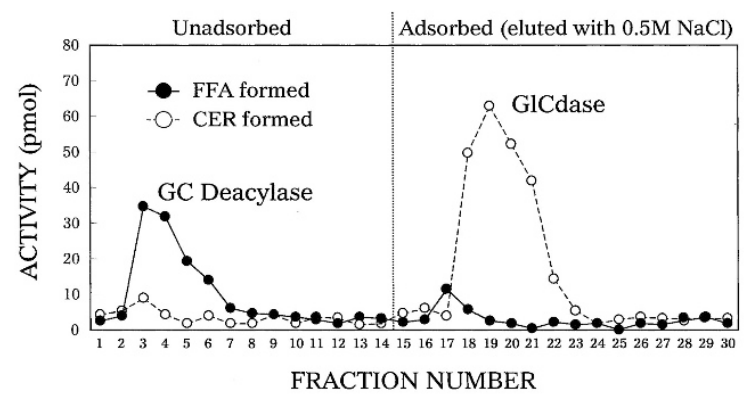

(B) GlCdase activity
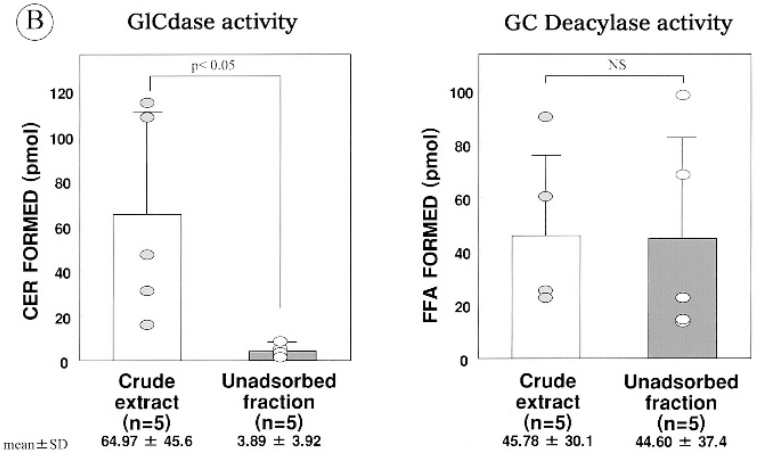

Figure 1

Distinction of glucosylceramide (GC) deacylase from $\beta$-glucocerebrosidase (GICdase). A, Chromatographic separation of GC deacylase and GICdase using extracts of the stratum corneum from patients with atopic dermatitis (AD). An extract of the stratum corneum was applied to CM-Sepharose equilibrated with elution buffer consisting of acetate buffer ( $\mathrm{pH} 5.0$ and $0.2 \mathrm{M} \mathrm{NaCl}$ ) to obtain $\mathrm{GC}$ deacylase as the pass-through fraction; the column was then eluted with 0.5 $\mathrm{M} \mathrm{NaCl}$ to yield GICdase. B, GC deacylase and GICdase activities of fractions separated by CM Sepharose. Enzymatic activities were evaluated for each eluted fraction according to the methods described in "Materials and Methods," and results are expressed as picomoles of free fatty acid (FFA) or ceramide (CER) released in the final or first upper phase per fraction.

\section{GICdase and GC Deacylase Activities in the Stratum Corneum}

Assays using [palmitic acid- ${ }^{14} \mathrm{C}$ ] GC as a substrate for GICdase (Fig. 2A) demonstrated that there were no significant differences in GICdase activity between the stratum corneum from uninvolved or involved skin of patients with $A D$ and from healthy controls. In contrast, assays using [palmitic acid- ${ }^{14} \mathrm{C}$ ] GC as a substrate for GC deacylase (Fig. 2B) revealed that the stratum corneum from involved forearm skin of patients with $A D$ has an extremely high level of $G C$ deacylase activity that is 5.4 times higher than that in healthy controls. In uninvolved skin with $A D, G C$ deacylase activity is still 2.8 times higher than that found in healthy controls. In contrast, the stratum corneum from patients with chronic eczema shows levels similar for both enzyme activities to healthy controls (Fig. 3).

\section{GC Deacylase in the Epidermis of Patients with AD}

Assays using [palmitic acid- ${ }^{14} \mathrm{C}$ ] GC as a substrate for GICdase (Fig. 4A) demonstrated that there was no significant difference in GICdase activity between the 

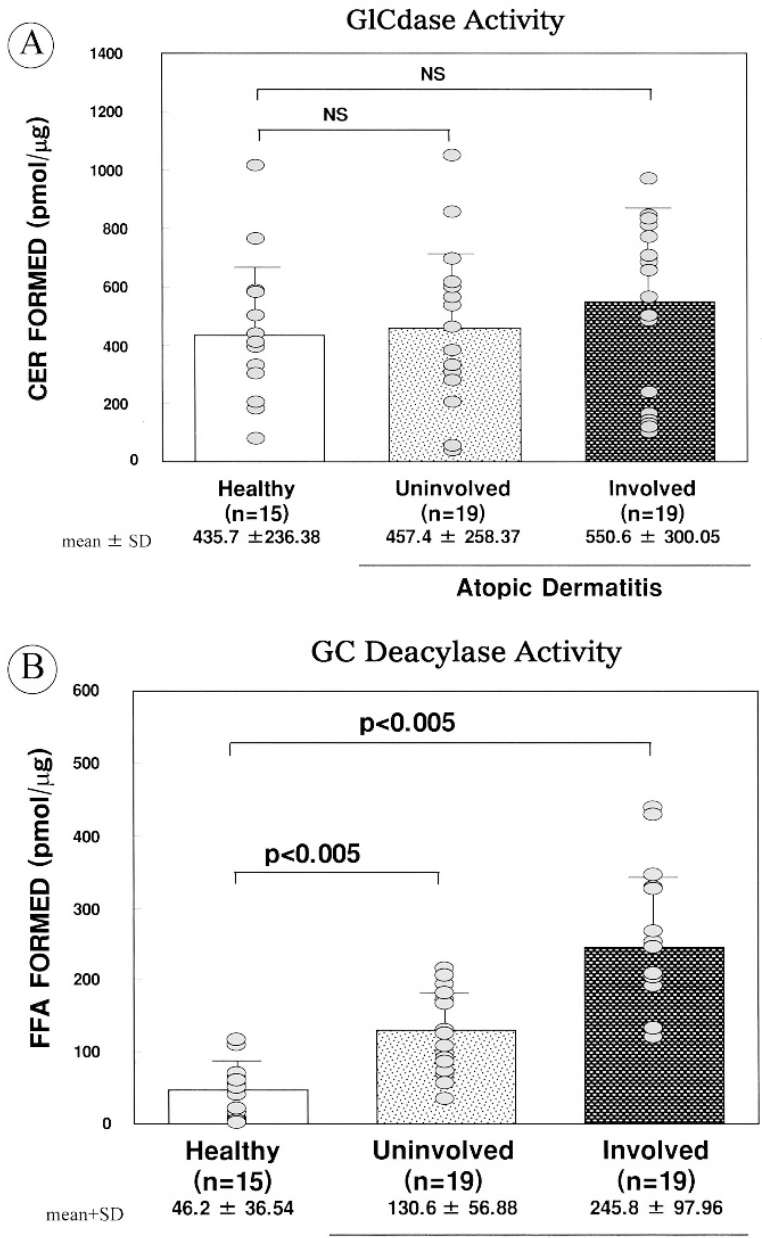

Atopic Dermatitis

Figure 2.

GICdase (A) and GC deacylase (B) activities in the stratum corneum from patients with $A D$ and healthy controls. A, GICdase activities are expressed as formed picomoles of radioactive CER into the first upper phase per microgram of protein after 16 hours of incubation at $\mathrm{pH} 4.7$ with $0.5 \mu \mathrm{Ci}$ [palmitic acid- $\left.{ }^{14} \mathrm{C}\right] \mathrm{GC}$. B, GC deacylase activities are expressed as liberated picomoles of radioactive FFA into the final upper phase per microgram of protein after 16 hours of incubation at pH 4.7 with $0.5 \mu \mathrm{Ci}$ [palmitoyl- $1-{ }^{14} \mathrm{C}$ ] glucosylsphingosine (GS).

epidermis of patients with $A D$ and healthy controls. However, assays using [palmitic acid $-{ }^{14} \mathrm{C}$ ] GC as a substrate for GC deacylase (Fig. 4B) demonstrated that the epidermis from lesional forearm skin from patients with $A D$ has a significantly higher level $(p<$ $0.005)$ of GC deacylase activity than does epidermis from healthy controls. In contrast, in the dermis, there were no significant differences in GICdase or GC deacylase activities between patients with $A D$ and healthy controls (Fig. 5).

\section{GS Is Significantly Increased in the Stratum Corneum of Patients with AD Compared with Healthy Controls}

Quantitative analysis of GS in the stratum corneum of patients with $A D$ revealed that there is a significant increase in the content of GS/mg stratum corneum (n $=90-100 ; p<0.001)$ in both the uninvolved and the involved stratum corneum of patients with $A D$ com-
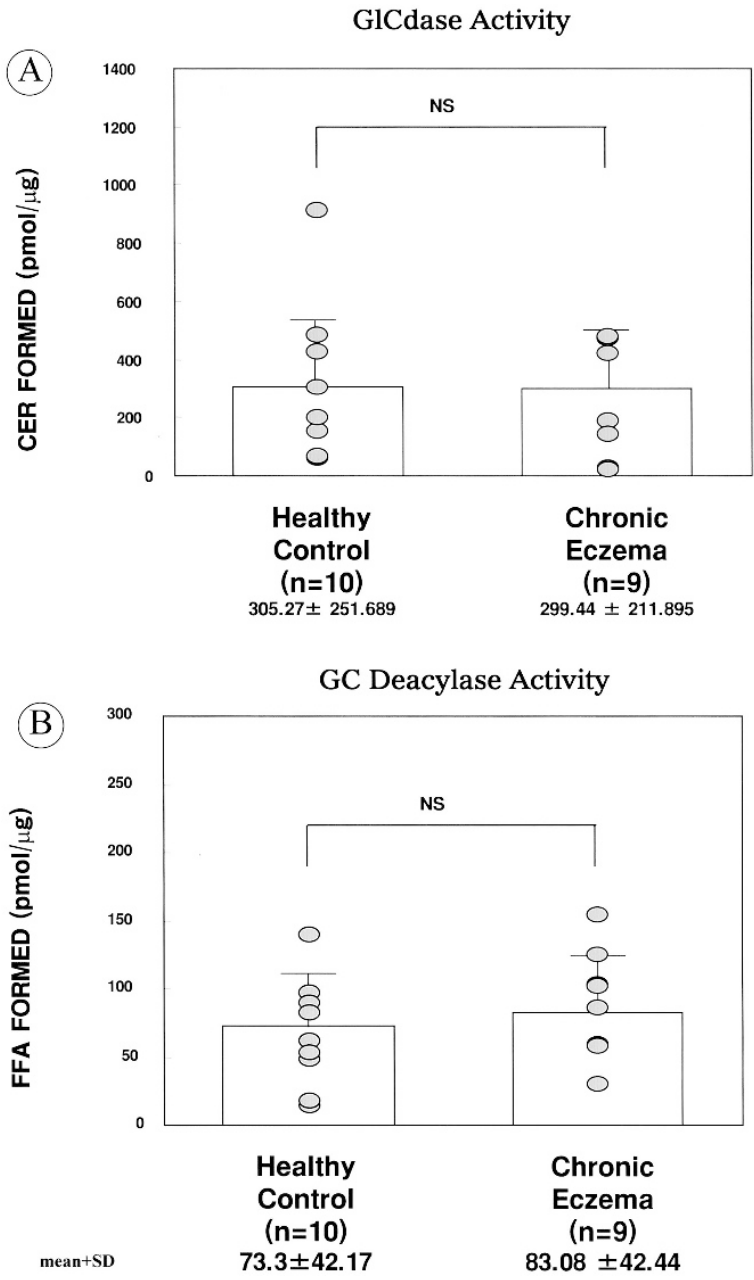

Figure 3.

GICdase (A) and GC deacylase (B) activities in the stratum corneum from patients with chronic eczema and healthy controls. A, GICdase activities are expressed as formed picomoles of radioactive CER into the first upper phase per microgram of protein after 16 hours of incubation at $\mathrm{pH} 4.7$ with $0.5 \mu \mathrm{Cl}$ [palmitic acid- ${ }^{14} \mathrm{C}$ ] GC. B, GC deacylase activities are expressed as liberated picomoles of radioactive FFA into the final upper phase per microgram of protein after 16 hours of incubation at $\mathrm{pH} 4.7$ with $0.5 \mu \mathrm{Ci}$ [palmitoyl- $1-{ }^{-14} \mathrm{C}$ ] GS.

pared with age-matched healthy controls (Fig. 6). Comparison of the amounts of total ceramides and ceramide-1 with GS in the same individuals (Fig. 7) demonstrated that there is a weak inverse relationship $(r=-0.416, p<0.05$ for total ceramides and $r=$ $-0.523, p<0.001$ for ceramide-1) between levels of ceramides and GS that accumulate in the stratum corneum. Among control, AD uninvolved, and involved groups, there is an inverse relationship between GS and total ceramides or ceramide- 1 only in the $A D$ involved group or in the $A D$ involved and uninvolved groups, respectively (Table 1). Comparison of total ceramides or ceramide-1 in the same individuals used for correlation analysis demonstrated that there is a significant decrease in the uninvolved and involved stratum corneum from patients with $A D$ compared with age-matched controls (Fig. 8). 


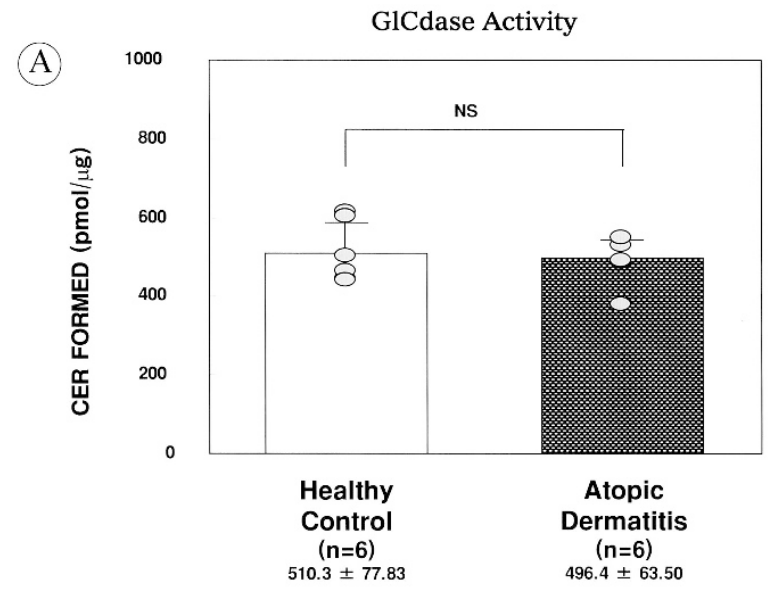

GC Deacylase Activity

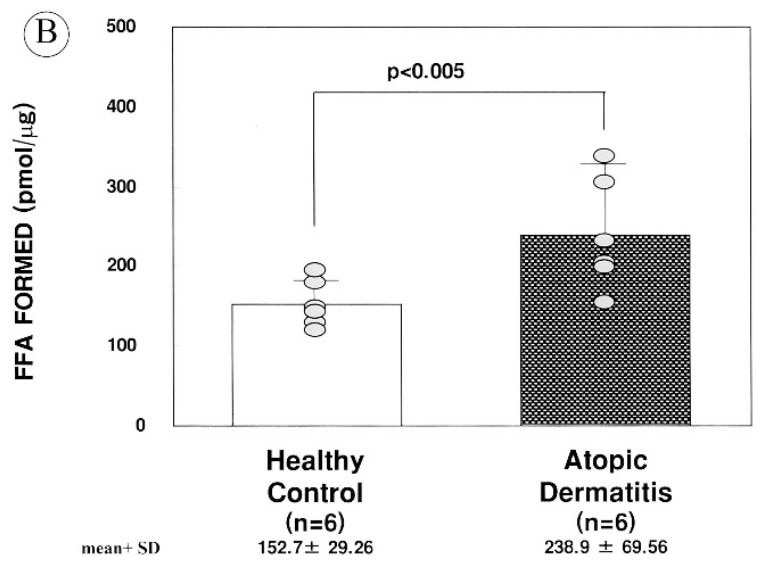

Figure 4.

GICdase (A) and GC deacylase (B) activities in the epidermis of patients with $A D$ and healthy controls. A, GICdase activities are expressed as formed picomoles of radioactive CER into the first upper phase per microgram of protein after 16 hours of incubation at $\mathrm{pH} 4.7$ with $0.5 \mu \mathrm{Ci}$ [palmitic acid- $\left.{ }^{14} \mathrm{C}\right] \mathrm{GC}$. B, GC deacylase activities are expressed as liberated picomoles of radioactive FFA into the final upper phase per microgram of protein after 16 hours of incubation at $\mathrm{pH} 4.7$ with $0.5 \mu \mathrm{Ci}$ [palmitoyl- $1-{ }^{14} \mathrm{C}$ ] $\mathrm{GS}$.

\section{Discussion}

Acylceramide is a unique species of the ceramide family that has been shown to be an essential component involved in barrier homeostasis because it is involved in forming the multilamellar membranous architecture in the intercellular spaces between the stratum corneum layers (Bouwstra et al, 1999). The importance of acylceramide in the maintenance of the barrier function is also supported by our previous evidence (Imokawa et al, 1994) that the topical application of pseudoacylceramide-containing ester-linked linoleic acid to the skin of essential fatty acid-deficient rats can restore the disturbed barrier function as revealed by the increased transepidermal water loss and epidermal hyperplasia. In skin with $A D$, we have consistently found that the acylceramide deficiency exists to the highest degree among several ceramide species, being predominantly attributable to the barrier disruption constitutively seen even in the nonlesional skin of patients with AD (Imokawa et al, 1991).
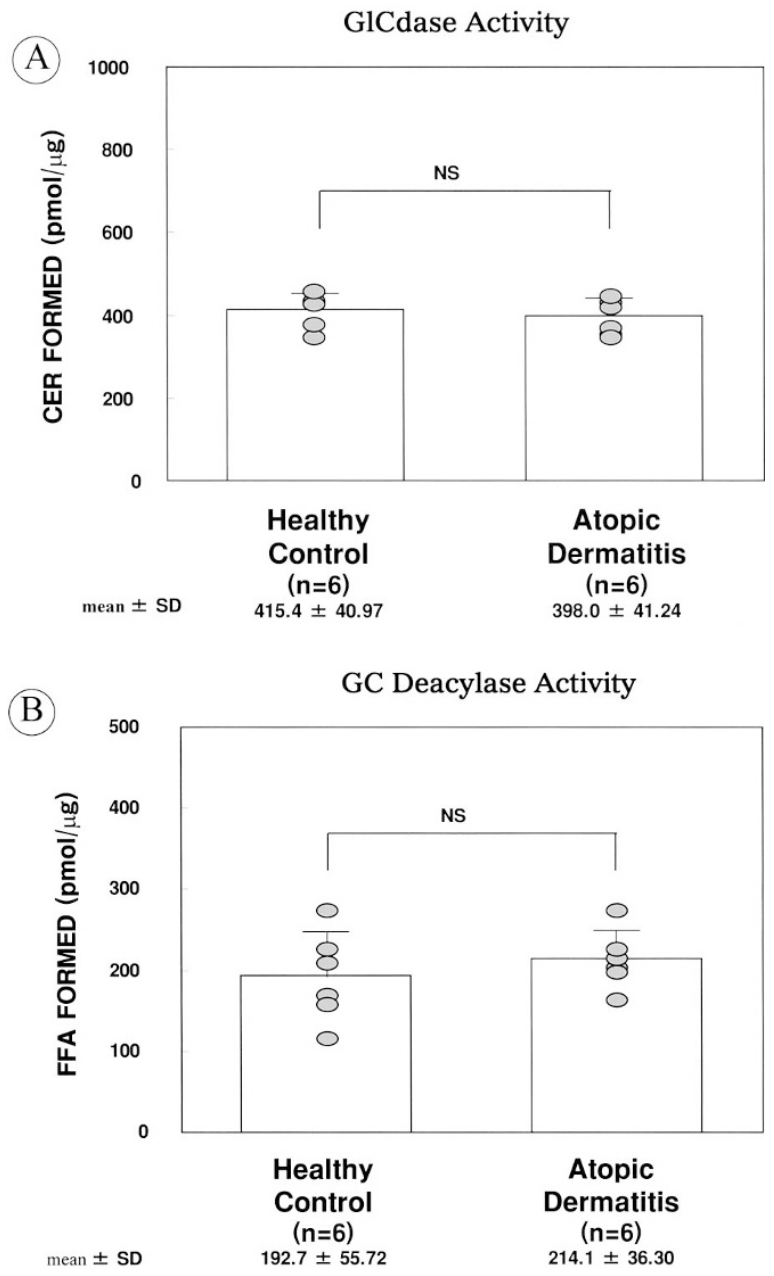

Figure 5.

GICdase (A) and GC deacylase (B) activities in the dermis of patients with $A D$ and healthy controls. A, GICdase activities are expressed as formed picomoles of radioactive CER into the first upper phase per milligram of protein after 16 hours of incubation at $\mathrm{pH} 4.7$ with $0.5 \mu \mathrm{Ci}$ [palmitic acid- ${ }^{14} \mathrm{C}$ ] GC. B, GC deacylase activities are expressed as liberated picomoles of radioactive FFA into the final upper phase per microgram of protein after 16 hours of incubation at $\mathrm{pH} 4.7$ with $0.5 \mu \mathrm{Ci}$ [palmitoyl- $-1{ }^{-14} \mathrm{C}$ ] GS.

The essential contribution of acylceramide to the disrupted barrier function in skin with $A D$ was also corroborated by the evidence that the topical application of pseudoacylceramide-containing ester-linked linoleic acid to the skin of patients with AD completely restored the skin barrier disruption with clinical improvement as revealed by the recovery of transepidermal water loss values to healthy control levels (Umeda et al, 1997). Because this barrier-disrupted situation in the clinically normal skin of patients with $A D$ may be responsible for the etiological background of $A D$ and because the observed deficiency of acylceramide is highly dependent on the barrier disruption found in skin with $A D$, it is of considerable importance to determine the biochemical mechanism(s) involved in the down-regulation of acylceramide production to elucidate the pathogenesis of $A D$ and its predisposition toward recurrent dermatitis.

As mechanisms underlying the deficiency of ordinary ceramides in skin with $A D$, we have already 


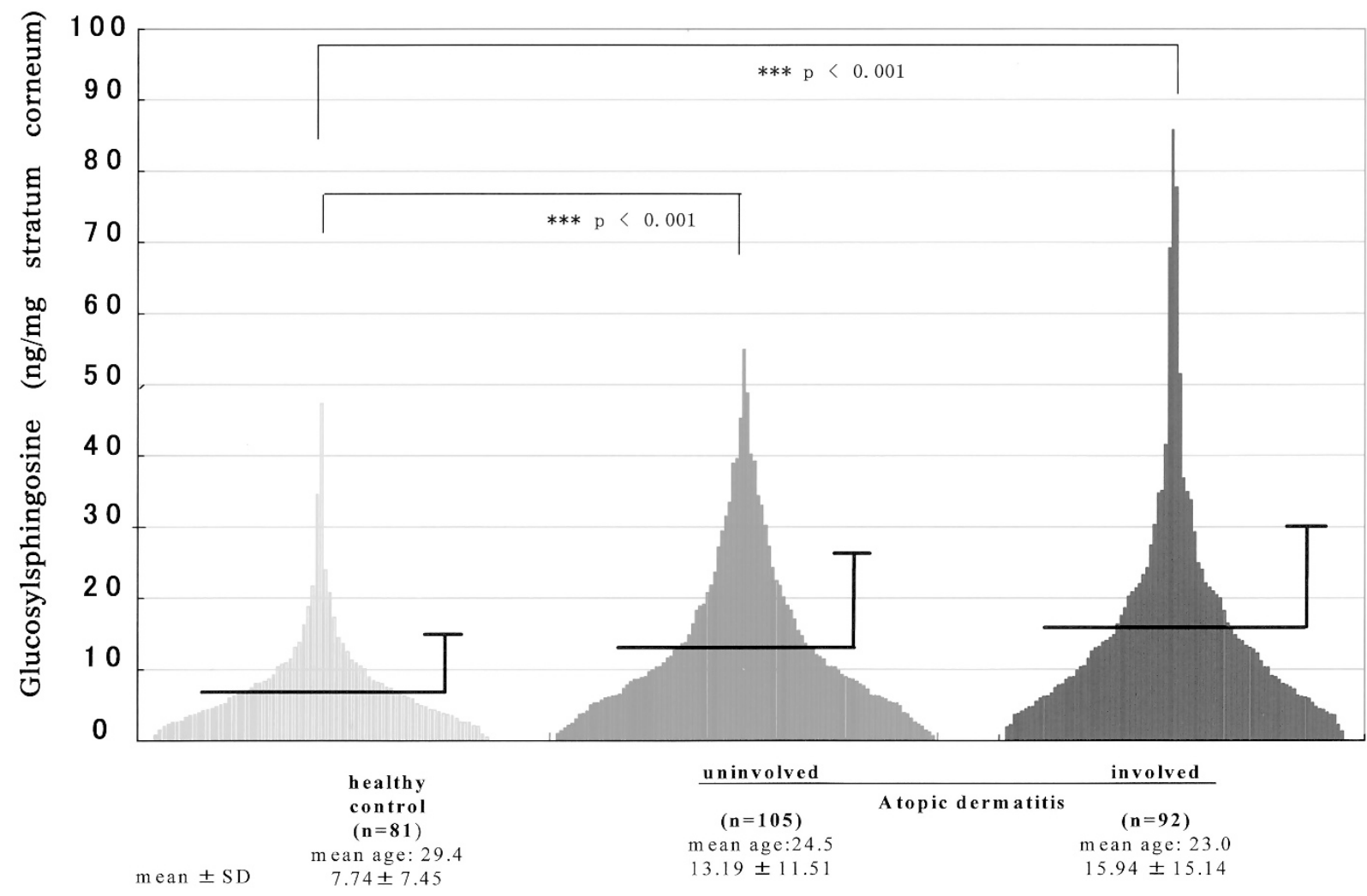

Figure 6.

The amount of GS in the upper stratum corneum from patients with AD and healthy controls. Age-matched controls: $n=81$, average 29.4 years of age; uninvolved skin of $A D: n=105$, average 24.5 years of age; involved skin of $A D: n=94$, average 23.0 years of age.

proposed (Fig. 9) that a novel sphingolipid metabolizing enzyme, termed SM deacylase, is highly expressed in the epidermis of patients with $A D$, which results in the interruption of ceramide production (Hara et al, 2000; Murata et al, 1996). This was based on evidence that there is no abnormality in the activities of other ceramide-generating or -degrading enzymes, including acid SMase (Hara et al, 2000; Jin et al, 1994). Thus, SM deacylase hydrolyzes SM at the acyl site to yield its lysoform sphingosylphosphorylcholine (SPC) instead of ceramide that is generated by acid SMase (Higuchi et al, 2000). The increased activity of this novel enzyme seems to be one possible biochemical mechanism leading to the ceramide deficiency in the lesional and in the nonlesional skin from patients with $A D$, because this enzyme is considered a bypass enzyme. Stimulation of SM deacylase by cutaneous inflammation leads to increased competition with the ordinary ceramide-producing enzyme acid SMase for the common substrate SM, which is attributable to the down-regulated levels of ceramides. In contrast, inflammation in the nonatopic normal skin in response to several stimuli, including ultraviolet light and scratching, tends to increase the biosynthesis of ceramides by enhancing the activities of ceramideproducing enzymes (Akimoto et al, 1993). As an alternative biochemical mechanism involved in the ceramide deficiency, Ohnishi et al (1999) proposed the involvement of bacteria that secrete CDase, which then degrades ceramide to generate sphingosine (SS).
This was based on evidence that the stratum corneum from patients with $A D$ contains a larger number of bacteria that produce CDase than the stratum corneum from healthy controls, when cultured in the presence of very specific nutrients such as ceramides (in which bacteria incapable of secreting CDase cannot survive) (Ohnishi et al, 1999). If this really occurs in vivo, then the total activity of acid or alkaline CDase in the stratum corneum (including bacteria secreting CDase) from patients with AD should be up-regulated compared with healthy controls, and levels of their reaction product, SS, should be increased in the stratum corneum from patients with $A D$. In the stratum corneum from patients with $A D$, we have already found that although there is no increase in alkaline CDase, the activity of acid CDase is significantly down-regulated compared with healthy controls (Arikawa et al, 2002). Consistent with this downregulation of acid CDase, its reaction product, SS, is significantly decreased in the stratum corneum of patients with $A D$ compared with healthy controls (Arikawa et al, 2002). Collectively, these observations indicate that there is no physiologic evidence for the up-regulation of ceramide degradation by CDase, suggesting that bacteria-derived CDase is not associated with the ceramide deficiency in the stratum corneum from patients with $A D$.

As novel properties of SM deacylase that are distinct from other deacylase enzymes reported by lto et al (1995), we have already characterized several enzy- 
(A)

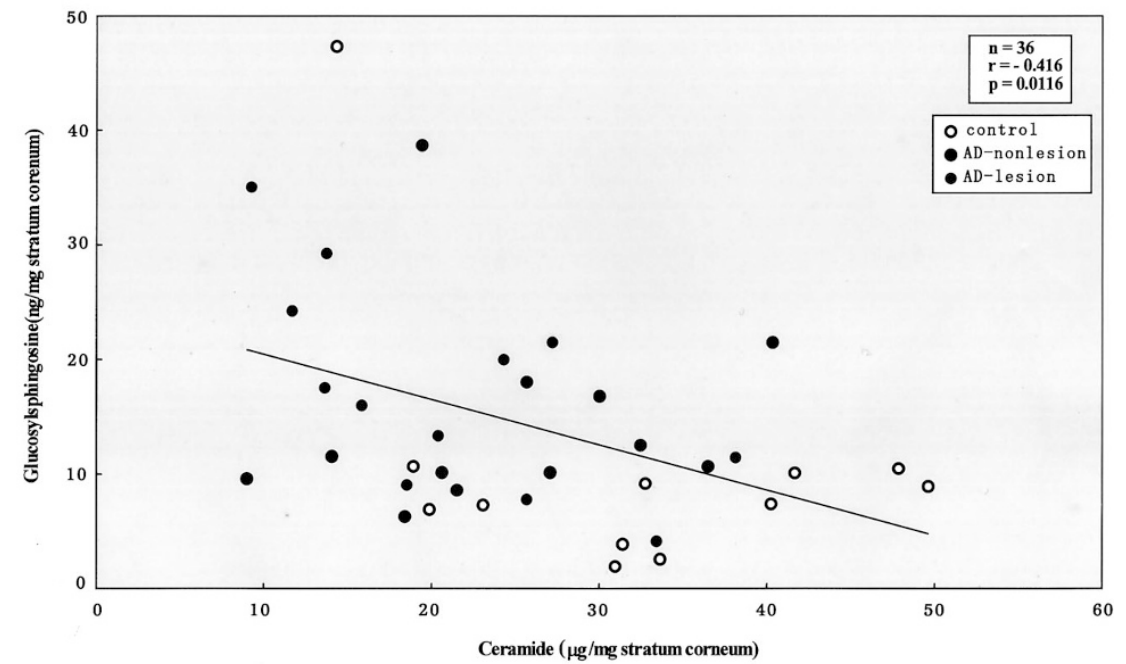

(B)

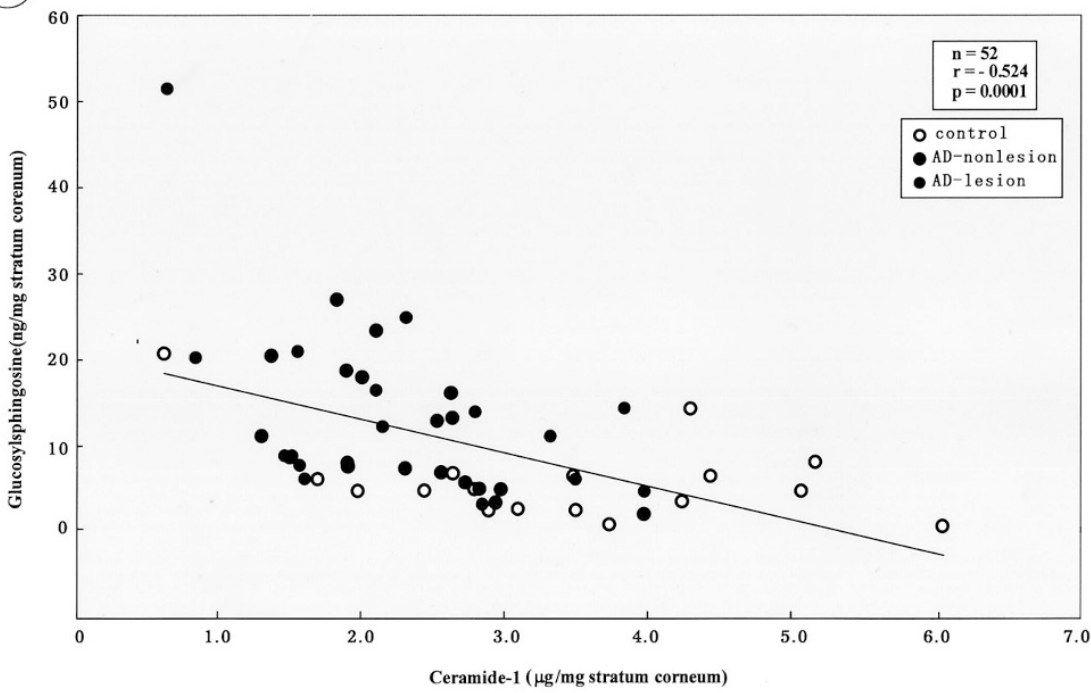

Figure 7.

Correlation between GS and CER in the upper stratum corneum from patients with AD and healthy controls. A, Total CER. Lesional skin of patients with AD: $n=12$; nonlesional skin of patients with $\mathrm{AD}: n=12$; healthy control skin: $n=13$. Total $n=36, \mathrm{r}=-0.416, p<0.05$. B, Ceramide-1(acylceramide). Lesional skin of patients with $\mathrm{AD}: n=16$; nonlesional skin of patients with AD: $n=19$; healthy control skin: $n=17$. Total $n=52, r=-0.523, p<0.0005$

Table 1. Relationship between GS and Ceramide-1 or Total Ceramide in Groups of Control, AD Uninvolved, and AD Involved

\begin{tabular}{ccccc}
\hline Counterpart to GS & Control & AD Uninvolved & AD Involved & Overall \\
\hline Ceramide-1 & $n=17$ & $n=19$ & $n=16$ & $n=52$ \\
& $r=-0.4062$ & $r=-0.5565$ & $r=-0.503946$ & $r=-0.52337$ \\
Total ceramide & $p=0.1056$ & $p=0.0133$ & $p=0.0466$ & $p=0.0001$ \\
& $n=12$ & $n=12$ & $n=12$ & $n=36$ \\
& $r=-0.4263$ & $r=0.1385$ & $r=-0.6733$ & $r=-0.41646$ \\
& $p=0.1669$ & $p=0.6678$ & $p=0.0164$ & $p=0.0116$ \\
\hline
\end{tabular}

GS, glucosylsphingosine.

matic characteristics of this enzyme as follows (Higuchi et al, 2000): (A) With the use of [palmitic acid-1$\left.{ }^{14} \mathrm{C}\right] \mathrm{SM}$ as a substrate, a sharp $\mathrm{pH}$ dependence was found for its catalytic activity, with a peak at $\mathrm{pH}$ 5.0. (B)
In contrast to the molecular weight of sphingolipid ceramide $N$-deacylase $(52,000)$ found in bacteria (Ito et al, 1995), preparative sodium dodecyl sulfatepolyacrylamide gel electrophoresis using AD epider- 


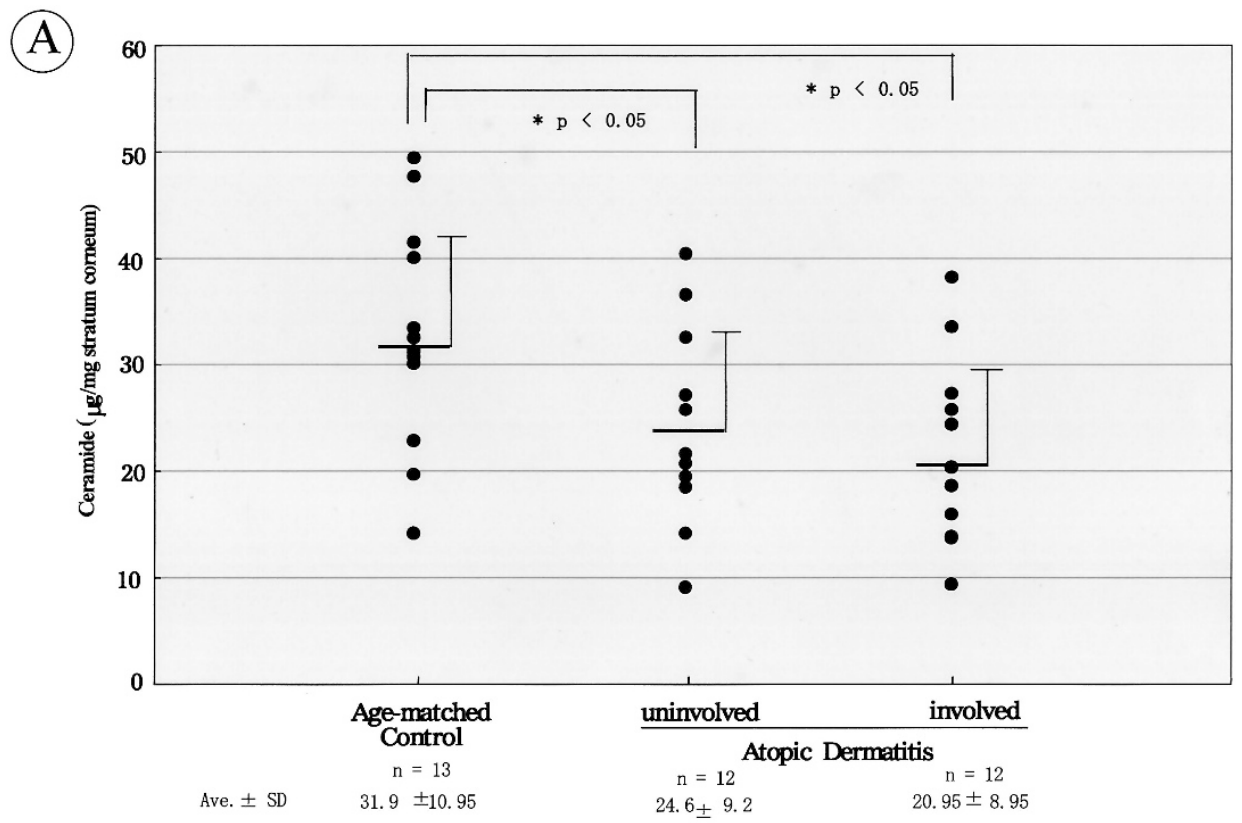

(B)

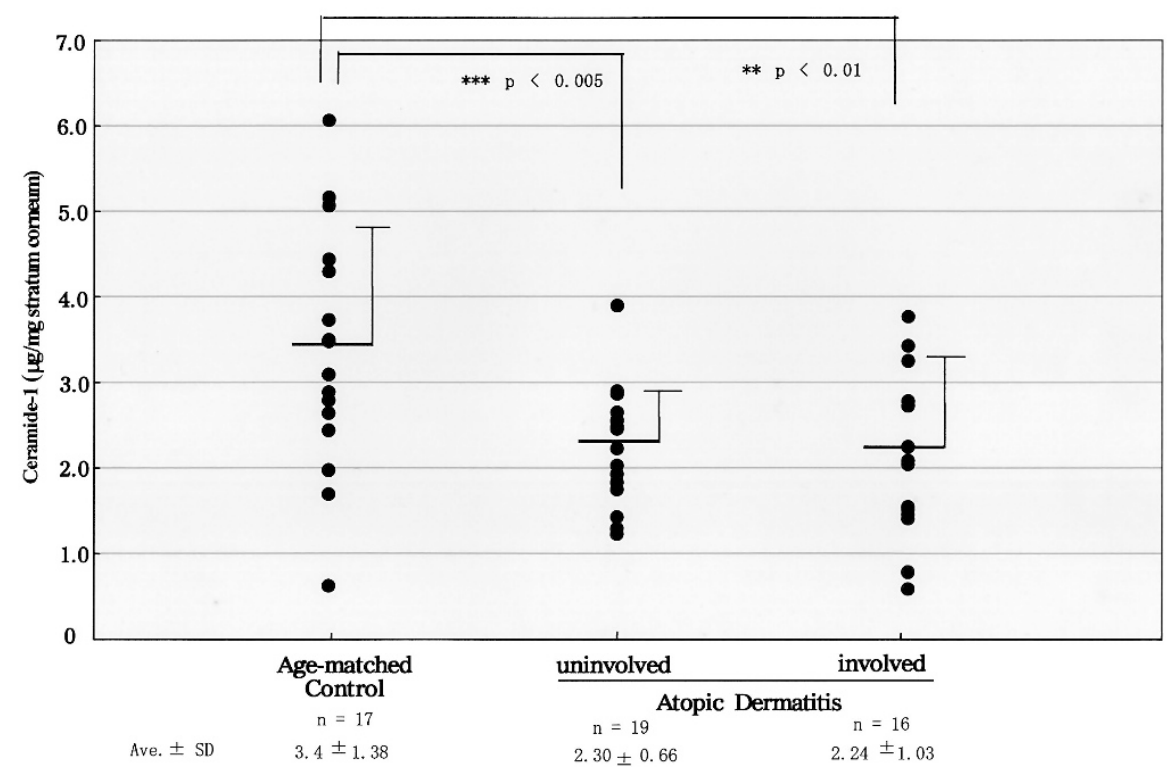

Figure 8.

The amount of total CER (A) and ceramide-1 (acylceramide) $(B)$ in the upper stratum corneum from patients with AD and healthy controls. The CER levels in the same individual used for correlation analysis were compared between $A D$ and control.

mis extracts revealed that the molecular weight of SM deacylase is 40,000 (Higuchi et al, 2000). (C) Analytical isoelectric focusing chromatography demonstrated that the $\mathrm{pl}$ values of SM deacylase, GICdase, SMase, and acid CDase were 4.2, 7.4, 7.0, and 5.7, respectively, again suggesting that these enzymes and sphingolipid ceramide $\mathrm{N}$-deacylase (with a pl $=6.9-$ 7.4) are different from each other. (D) Enzymatic analysis using the pl 4.2 protein partially purified by isoelectric focusing, which contained no contamination with acid CDase, GICdase, or SMase, showed that radiolabeled SPC was enzymatically liberated from [choline-methyl- ${ }^{14} \mathrm{C}$ ]SM used as a substrate. (E) The $\mathrm{pl} 4.2$ protein purified from the stratum corneum extract of patients with AD could hydrolyze [N-palmitoyl-1- $\left.{ }^{14} \mathrm{C}\right] \mathrm{SM}$ but not [N-palmitoyl-1- $\left.{ }^{14} \mathrm{C}\right]$ ceramide, thus indicating that the SM deacylase is distinct from any known CDases or deacylases.

Consistent with the high expression of SM deacylase, we also found that the metabolic intermediate SPC accumulates in the upper stratum corneum of patients with AD (Fig. 9). In contrast, in patients with chronic eczema or contact dermatitis, there was no significant up-regulation in the activity of SM deacylase (Hara et al, 2000; Murata et al, 1996) or in the content of SPC in the stratum corneum compared with healthy controls (Okamoto et al, 1999), thus indicating that the up-regulated activity of SM deacylase is not a 


\section{Basal Layer}

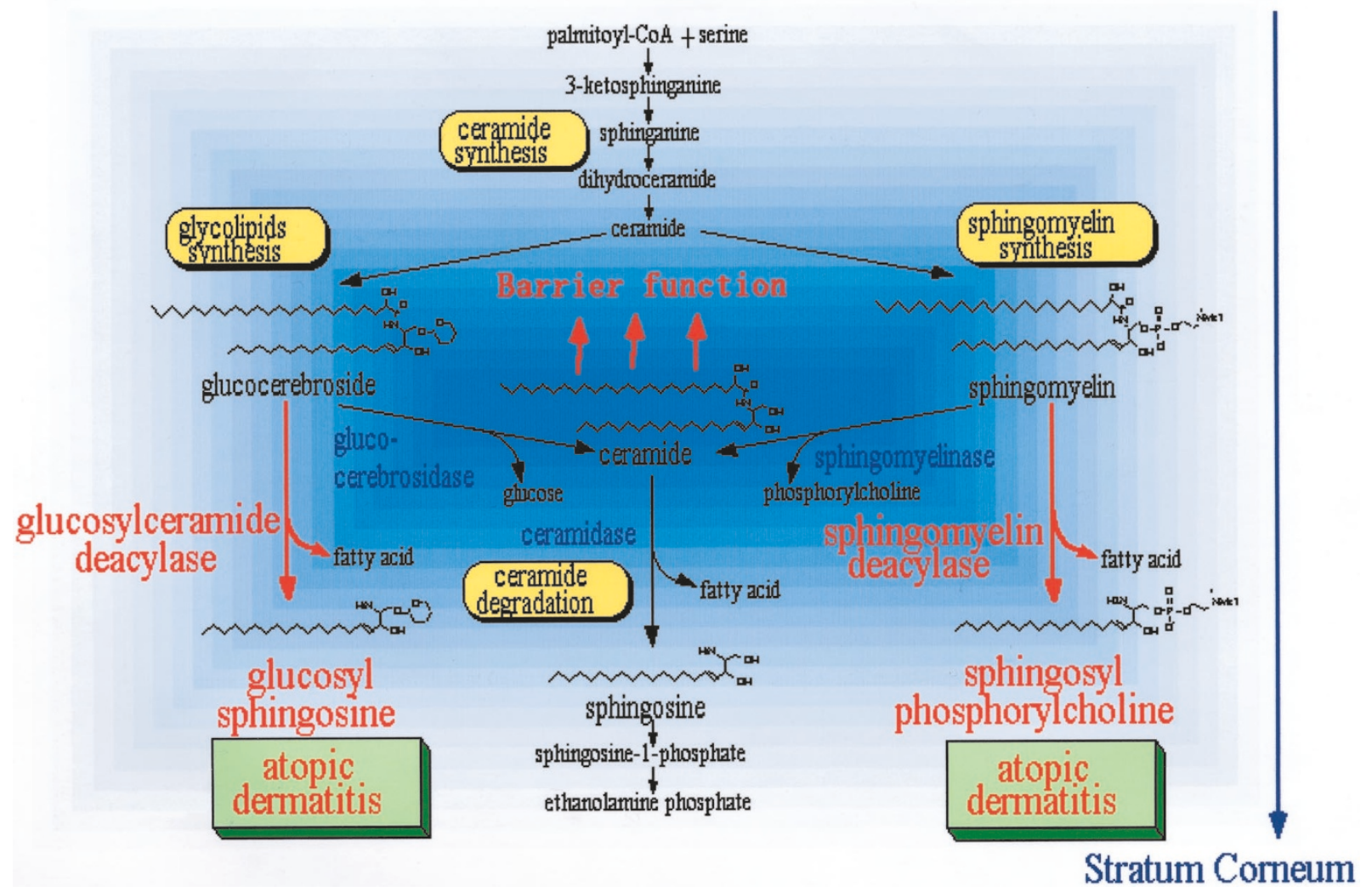

Figure 9.

Altered sphingolipid metabolisms discovered in the AD epidermis.

simple result of cutaneous inflammation but probably is specific to skin from patients with AD.

Recently, it was reported that omega-OH ceramide, an important acylceramide precursor in the mammalian stratum corneum, does not derive from SM, which suggests that these species derive solely from GC precursors (Uchida et al, 2000). Thus, it is likely that acylceramides (ceramides 1 or 4) are synthesized not by hydrolysis of SM but through pathways involving deglucosylation of acylglucosylceramides by GICdase. On the basis of these findings, the acylceramide deficiency observed in AD (Imokawa et al, 1991) could not be explained in terms of the up-regulation of SM deacylase. In this regard, we supposed the possibility that if, like SM deacylase, an unknown enzyme tentatively termed GC deacylase, capable of catalyzing $\mathrm{N}$-deacylation of $\mathrm{GC}$, is highly expressed in the epidermis of patients with $A D$, then this abnormality may lead to the acylceramide deficiency. In this study, we used cationic ion exchange chromatography to separate GC deacylase from GICdase, and we used [palmitic acid- ${ }^{14} \mathrm{C}$ ] GC as a substrate to show that the stratum corneum extract from patients with $A D$ has the potential to hydrolyze GC directly at the N-acyl site to release ${ }^{14} \mathrm{C}$-labeled free fatty acid. Comparison of the activity of GC deacylase revealed that there are significant increases in its activity in the involved (5.4-fold increase) and uninvolved (2.5-fold increase) stratum corneum or in the involved (3-fold increase) epidermis from patients with $A D$ compared with healthy controls. In contrast, there is no difference in the activity of GICdase in the skin of patients with AD and healthy controls, which is consistent with our previous reports (Jin et al, 1994). Interestingly, in the dermis, there was no difference in the activity of GC deacylase between patients with $A D$ and healthy controls, which suggests that the abnormality of sphingolipid metabolism in AD may be confined to the epidermis. A higher activity of the GC deacylase in the dermis than in the stratum corneum from healthy controls may reflect the possibility that a defect in down-regulation of its activity in the epidermis is associated with AD. Collectively, our observation suggests that the acylceramide deficiency in $A D$ is mainly attributable to the accentuated activity of GC deacylase (Fig. 9). Thus, the abnormally expressed GC deacylase hydrolyzes (acyl) GC at the N-acyl site to yield its lysoform, GS, instead of the formation of (acyl)ceramides by GICdase. The enhanced activity of this novel enzyme is proportional to the diminished level of (acyl)ceramide because of the enzymatic competition toward the same substrate, GC, between GC deacylase and GICdase.

To characterize the physiological and functional relevance of GC deacylase to the acylceramide deficiency in the epidermis of patients with $A D$, it was important to determine whether its enzymatic reaction product, GS, is released into the epidermis and accu- 
mulates in the stratum corneum. The present quantitative analysis of GS in the upper stratum corneum of patients with $A D$ demonstrates that there is a significant up-regulation in the amount of GS in uninvolved and in involved stratum corneum from patients with $\mathrm{AD}$ compared with age-matched healthy controls. Taken together with the increased activity of GC deacylase in $A D$, our results suggest that the upregulation of GS is associated with the altered lipid metabolism characteristic for AD (Fig. 9).

A similar accumulation of reaction products by corresponding N-deacylase enzymes has been found in Gaucher disease (Fig. 10), in which there is an accumulation of GS as a result of a defect of GICdase activity (Orbisky et al, 2000). In Gaucher disease, there has been no explanation for the accumulation of GS in terms of the action of the corresponding $\mathrm{N}$-deacylase, namely GC deacylase. Thus, GS accumulation has also been speculated to result from a defect of GICdase because GS can serve as a substrate for that enzyme (Orbisky et al, 2000). Similarly, the possible existence and expression of GC deacylase in Gaucher disease would provide a reasonable mechanism for the up-regulation of GS. Another similar accumulation of substrates and reaction products by corresponding $\mathrm{N}$-deacylase enzymes has been found in NiemannPick disease (Fig. 10), in which defects of SMase have been associated and that results in the lipidosis for SM, eliciting an accumulation of SPC (Schmuth et al, 2000), although no data suggest that this SPC accumulation is linked to the expression of an SM deacylase-like enzyme. Another similar relevance of $\mathrm{N}$-deacylase for the generation of psychosine has been reported in globoid cell leukodystrophy or Krabbe's disease (Kanazawa et al, 2000). The primary defect of globoid cell leukodystrophy is a deficiency in galactosylceramidase activity, which leads to the accumulation of galactosylceramide and its metabolic intermediate, galactosylsphingosine. This was speculated to be produced by deacylation of galactosylceramide (Kanazawa et al, 2000), although there is no evidence for the expression of galactosylceramide deacylase in globoid cell leukodystrophy. Such altered lipid metabolisms associated with genetic defects, which lead to the accumulation of lipid substrates and deacylated metabolic intermediates, strongly suggest the principle that defects of metabolic enzymes might induce corresponding alternative pathways in which those substrates are converted to corresponding lysoforms by deacylation. Such a possible induction of an alternative pathway after the loss of metabolic enzymes has been reported in a Gaucher-like mouse induced by a glucosylceramidase inhibitor that shows the accumulation of GS in tissue (Atsumi et al, 1993). Such a similarity in the accumulation of lysosphingolipids as bioproducts by $\mathrm{N}$-deacylases between $\mathrm{AD}$ and Gaucher or Niemann-Pick diseases seems to suggest the possibility that in the skin with $A D$, somewhat unveiled biological influences, such as the deterioration of SMase or GICdase enzymes, may induce expression of such bypass enzymes under the unknown conditions associated with atopic diathesis,

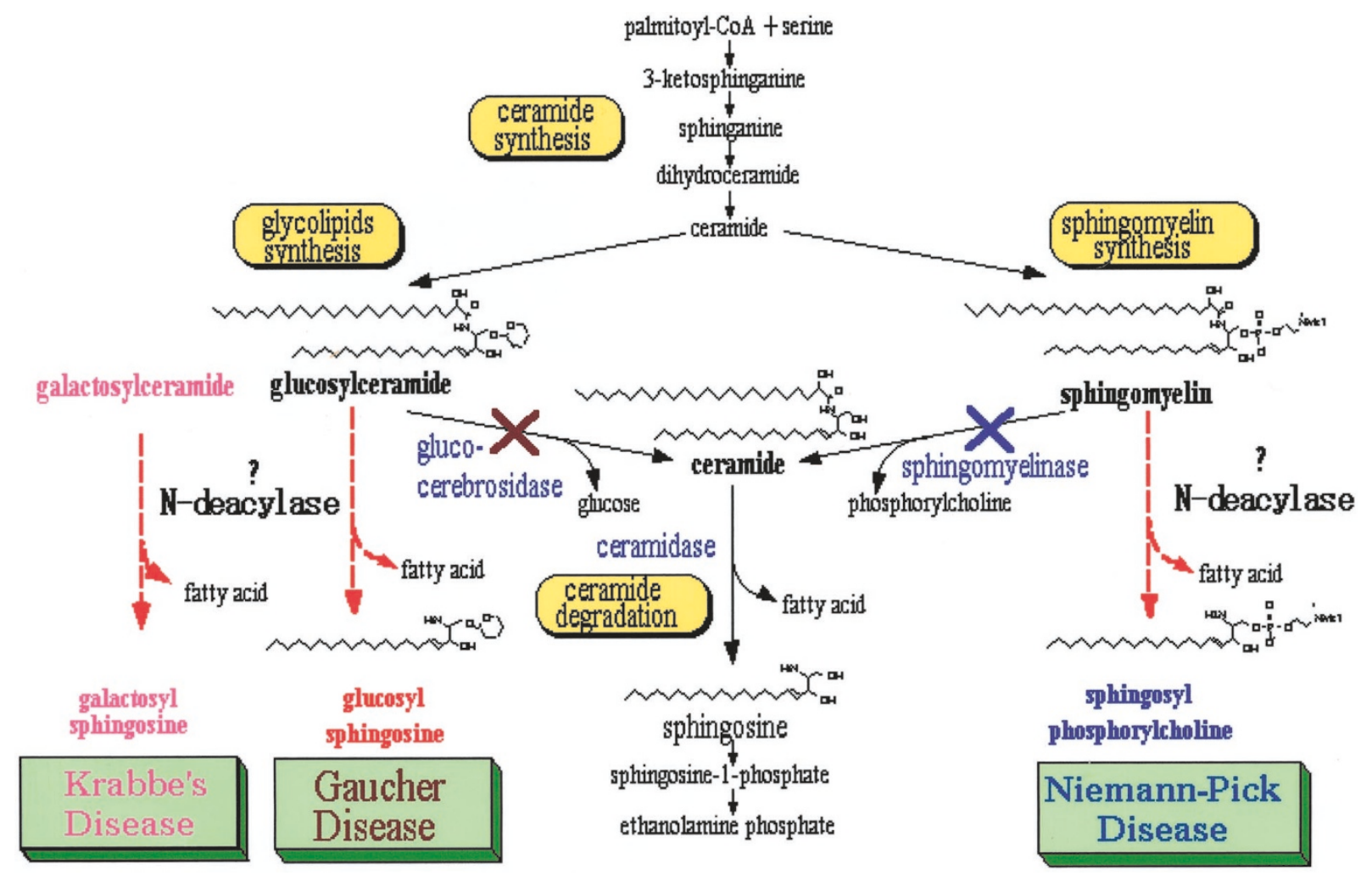

Figure 10.

Altered sphingolipid metabolisms discovered in Gaucher or Niemann-Pick diseases. 
although additional studies will be required to support such a hypothesis.

In conclusion, we have elucidated the physiologic and functional relevance of GC deacylase to the acylceramide deficiency that is an essential causative factor in the barrier-disrupted skin of patients with AD. We found that extracts of the stratum corneum or the epidermis from patients with $A D$ have a higher potential to hydrolyze $\mathrm{GC}$ at the $\mathrm{N}$-acyl site to release ${ }^{14} \mathrm{C}$-labeled free fatty acid than those from healthy controls. Interestingly, the metabolic intermediate, GS, which is an essential surrogate to determine whether GC deacylase is functioning in situ in the epidermis, is significantly up-regulated in the uninvolved and in the involved stratum corneum of patients with $A D$ compared with healthy controls. Collectively, our results suggest that the novel epidermal enzyme, GC deacylase, is expressed in situ at significant levels in the epidermis of patients with AD. This results in the production and accumulation of GS, which leads in turn to the acylceramide deficiency seen in the stratum corneum of those patients. It is likely that the biogenesis of this highly expressed GC deacylase may be relevant to the atopic diathesis, if AD could be considered as an acylceramide-deficient disease.

\section{Materials and Methods}

\section{Preparation of Stratum Corneum and Epidermis Extracts}

Diagnoses of AD were made according to the criteria of Hanifin and Rajka (1989). Patients and healthy individuals were asked to clean the skin with soap in the morning of the date when sampling was carried out and not to apply any materials on the forearm skin before tape stripping in the hospital. Specimens of stratum corneum were obtained from normal forearm skin of healthy individuals (aged from 18 to 31 years; mean age, 28.2 years) and from uninvolved and involved skin of patients with AD (aged from 14 to 33 years; mean age, 21.7 years) by tape-stripping three times (Protect-label/B type, 40-60 mm, luchiseieidou Corporation, Japan) in the same region. Such tapes were stored frozen at $-80^{\circ} \mathrm{C}$ until used. Informed consent was obtained from each patient before sampling.

For measurement of GC deacylase activity, the three tapes were immersed in $40 \mathrm{ml}$ of $50 \mathrm{~mm}$ acetate buffer ( $\mathrm{pH} 4.7$ ), containing $0.5 \mathrm{~mm}$ phenylmethylsulfonyl fluoride and $0.1 \%$ Triton $\mathrm{X}-100$ for 30 minutes at $4^{\circ} \mathrm{C}$, and were then sonicated on ice for a total of 3 minutes with 30-second bursts in a Bioruptor sonicator (Olympus Corporation). Mixtures containing the three tapes were then further incubated in extraction buffer overnight at $4^{\circ} \mathrm{C}$ to obtain Triton $\mathrm{X}-100$ extracts for subsequent assay. After the tapes were removed, the extracts were concentrated to approximately 0.5 $\mathrm{ml}$ using a Centriprep 10 and Centricon 10 (Amicon, Millipore Corporation, Bedford, MA), and the concentrated solutions were used for the GC deacylase assay.
For GC deacylase assays of the epidermis, 4-mm skin punch biopsy specimens were treated with 1000 unit/ml dispase at $4^{\circ} \mathrm{C}$ for 4 hours to separate the epidermis. Each isolated epidermal sheet was homogenized with a glass homogenizer in $300 \mu \mathrm{l}$ of $50 \mathrm{~mm}$ acetate buffer ( $\mathrm{pH} 4.7)$, containing $0.5 \mathrm{~mm}$ phenylmethylsulfonyl fluoride. The homogenates were centrifuged at $10000 \times g$ for 30 minutes to obtain supernatant and particulate fractions. The latter was further solubilized for 2 hours in $300 \mu$ l of $50 \mathrm{~mm}$ acetate buffer ( $\mathrm{pH} 4.7$ ), containing $0.5 \mathrm{~mm}$ phenylmethylsulfonyl fluoride and $0.1 \%$ Triton $\mathrm{X}-100$. The solubilized mixture was then centrifuged at $10000 \times g$ for 30 minutes to obtain a supernatant of the extracted fraction, which was used for the GC deacylase assay as detailed below.

\section{Cationic Exchange Chromatography Using CM Sepharose}

The crude stratum corneum extract $(400 \mu \mathrm{l})$ was applied and eluted on a CM Sepharose column (1 ml volume) equilibrated in $0.2 \mathrm{M}$ acetate buffer ( $\mathrm{pH} 5.0)$, containing $0.1 \%$ Triton $\mathrm{X}-100$ (Buffer A). The passthrough fraction $(400 \mu \mathrm{l})$ and the eluted fraction $(400 \mu \mathrm{l}$ each) after loading in $0.5 \mathrm{M} \mathrm{NaCl}+$ Buffer A were collected and assayed using $200 \mu \mathrm{l}$ each for GC deacylase and GICdase activities. Pooled fractions (20 $\mathrm{ml}$ ) were also condensed to $1 \mathrm{ml}$ and assayed using $200 \mu \mathrm{l}$ for the same enzyme activities.

\section{Measurement of GC Deacylase and GICdase Activities}

GC deacylase and GICdase activities were assayed by measuring the amount of radioactive palmitic acid and ceramide released from $\mathrm{N}$-[palmitoyl- $\left.1-{ }^{14} \mathrm{C}\right]$ GS (47 $\mathrm{mCi} / \mathrm{mmol})$, which was synthesized using $\left[1-{ }^{14} \mathrm{C}\right]$ palmitic acid (55 mCi/mmol; Amersham, Bucks, UK) according to the method described previously (Higuchi et al, 2000). The standard reaction mixture (final volume, $300 \mu \mathrm{l})$ contained $50 \mathrm{~mm}$ acetate buffer $(\mathrm{pH}$ 4.7), $10 \mathrm{nmol} 0.5 \mathrm{mCi}$ [palmitoyl-1- ${ }^{14} \mathrm{C}$ ] GS, $0.1 \%$ Triton $\mathrm{X}-100,20 \mathrm{~mm} \mathrm{CaCl}_{2}$, and tissue extracts. The reaction mixture was incubated for 16 hours at $37^{\circ} \mathrm{C}$ and was terminated by the addition of $50 \mu \mathrm{l}$ of $2 \mathrm{mg} / \mathrm{ml}$ carrier palmitic acid, followed by $3.0 \mathrm{ml}$ of Dole's reagent (2-propanol-heptane-1 $\mathrm{N} \mathrm{NaOH} \mathrm{40:10:1} \mathrm{vol/}$ vol). After $1.8 \mathrm{ml}$ of heptane and $1.6 \mathrm{ml}$ of water were added, the mixture was vortex-mixed and centrifuged for 5 minutes at 2,000 $\times$ g. One milliliter of the upper heptane phase, which contained the enzymatically released radioactive ceramide, was transferred to a vial and was mixed with scintillation fluid. The radioactivity was determined in a liquid scintillation counter to assess GC deacylase activity. The remainder of the heptane phase was carefully aspirated, and the lower phase was washed twice with $2 \mathrm{ml}$ of heptane. Thereafter, $1 \mathrm{ml}$ of $1 \mathrm{~N} \mathrm{H}_{2} \mathrm{SO}_{4}$ and $2.4 \mathrm{ml}$ of heptane were added, and the mixture was vortex-mixed, then centrifuged for 10 minutes at $2,000 \times g$. One milliliter of the upper phase was washed with an equal volume of $1 \mathrm{~N} \mathrm{H}_{2} \mathrm{SO}_{4}$ to remove the small amount of unreacted GC. After mixing and centrifugation, $0.8 \mathrm{ml}$ of the 
washed upper phase was counted by liquid scintillation to measure GC deacylase activity. For establishing the background, blank tubes were carried through without the enzyme source. The labeled reaction products from the GC deacylase and GICdase activities were then identified by thin-layer chromatography (TLC) on silica gel with the solvent system chloroformmethanol-acetic acid 94:1:5 (vol/vol). These were compared with radioactive standards, SM, ceramide (ie, palmitoylsphingosine), which was prepared as described previously (Yada et al, 1995). Radio- or normal TLC analysis demonstrated that reaction products in the first upper heptane phase (which partitioned in the biphasic system of Dole) and in the final upper phase (after washes with $\mathrm{H}_{2} \mathrm{SO}_{4}$ ) were ceramide and palmitic acid, respectively. These had mobilities on TLC plates nearly identical to labeled standards, indicating an effective separation of palmitoylsphingosine (ceramide) and palmitic acid using this partition method. The activities of GC deacylase and GICdase were expressed as picomoles of ceramide or palmitic acid released in the first or final upper phase per microgram of protein.

\section{Lipid Extraction}

For measuring ceramide and GS, stratum corneum lipids were extracted with the modified method of Rawlings et al (1996). In brief, stratum corneum was removed from the volar side of forearm skin by stripping with adhesive tape (P.P.S. Nichiban, Tokyo, Japan) three times. The stratum corneum was then separated from the adhesive tape by washing with n-hexane using ultrasonication (Bransonic B3200; Yamato Scientific Company, LTD.). Sphingolipids, including ceramides and GS, were extracted from the separated stratum corneum with chloroform/methanol (2/1) and were subjected to quantification of ceramides and GS.

\section{Quantitative Assay for GS by N-Acetylation with $\left[{ }^{14} \mathrm{C}\right]$-Acetic Anhydride}

GS was quantified using the modified method of Yatomi et al (1995). The dried samples were dissolved in $20 \mu$ l of $0.008 \mathrm{~N} \mathrm{NaOH}$ in dehydrated methanol. The acetylation reaction as depicted in Figure $11 \mathrm{~A}$ was started by adding $20 \mu \mathrm{l}$ of $10 \mathrm{~mm}$ [methyl- ${ }^{14} \mathrm{C}$ ]-acetic anhydride (Amersham) in chloroform and was allowed to proceed for 2 hours at $37^{\circ} \mathrm{C}$. The remaining anhydride was hydrolyzed by the addition of $200 \mu \mathrm{l}$ of 0.2 $\mathrm{N} \mathrm{NaOH}$ in methanol and incubation at room temperature. The $\left[{ }^{14} \mathrm{C}\right]-\mathrm{C}_{2}-\mathrm{GC}$ produced was extracted by adding $0.78 \mathrm{ml}$ of methanol, $0.98 \mathrm{ml}$ of chloroform, and $0.9 \mathrm{ml}$ of $1 \mathrm{~N} \mathrm{KCl}$. Carrier lipids were applied to the solvent to increase the efficiency of extraction. The resultant lower phase was evaporated under $\mathrm{N}_{2}$ and was then resuspended in a small volume of chloroform/methanol (2:1) and applied to silica gel 60 HPTLC plates (Merck). The plates were developed in chloroform/methanol/acetone (76:20:4) in a horizontal HPLC chamber (CAMAG; Muttenz, Switzerland) to resolve
(A)

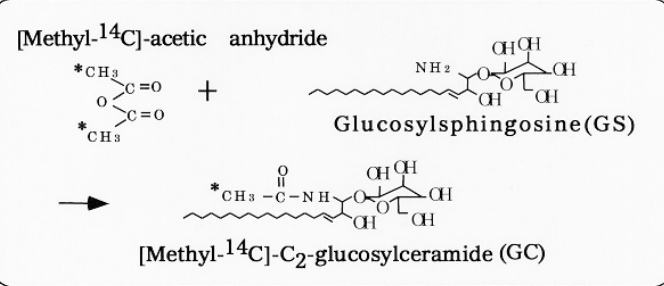

(B)

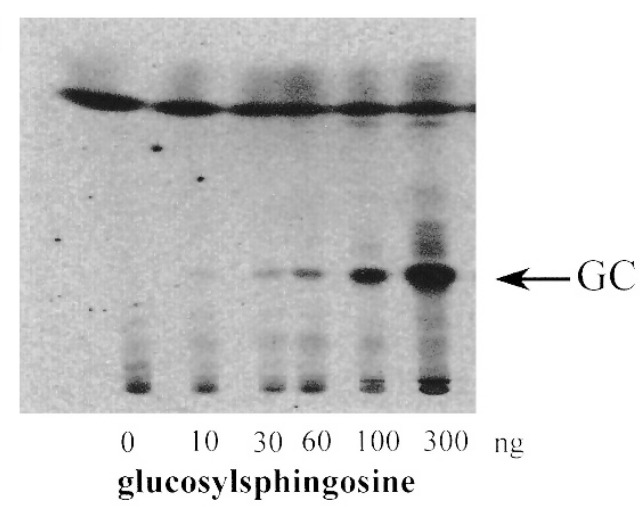

(C)

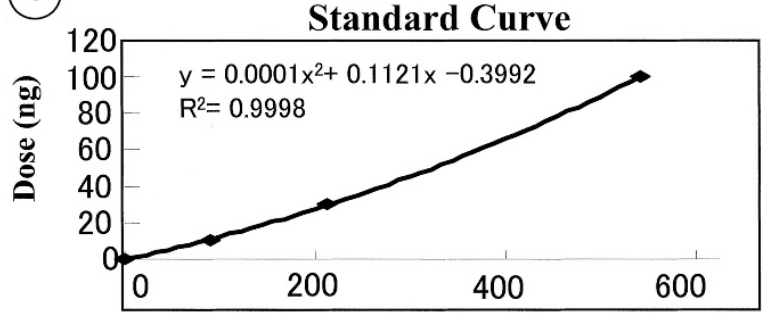

Count

Figure 11.

Quantitation of GS by reaction with $\left[{ }^{14} \mathrm{C}\right]$-acetic anhydride. A, Chemical reaction of GS with $\left[{ }^{14} \mathrm{C}\right]$-acetic anhydride. B, TLC separation of ${ }^{14} \mathrm{C}-\mathrm{C}_{2}$ GS produced. C, The standard curve of GS for quantification.

C-2 glucosylceramide and were quantified using a Bioimaging analyzer (BAS-2000; Fuji Film Corporation, Japan). Representative chromatograms of $\left[{ }^{14} \mathrm{C}\right]-\mathrm{C}_{2}{ }^{-}$ GC and the standard GS curve are shown in Figure 11, $\mathrm{B}$ and $\mathrm{C}$, respectively.

\section{TLC}

TLC were developed twice with chloroform:methanol:acetic acid (190:9:1) to resolve ceramide, cholesterol, cholesterol ester, triglyceride, free fatty acid, and cholesterol sulfate. After solvent development, the chromatograms were air-dried, sprayed with $10 \%$ $\mathrm{CuSO}_{3}$ and $8 \% \mathrm{H}_{3} \mathrm{PO}_{4}$ in aqueous solution, and then charred on a $180^{\circ} \mathrm{C}$ hot plate. The charred lipids were quantified by photodensitometry (Shimazu CS-9000), and the data were subjected to a 2-Dimensional Image Analyzer (Shimazu). Ceramides were quantified by determining micrograms of ceramides on a TLC chart from appropriate commercial standards and are expressed as micrograms of ceramide per milligram of stratum corneum weight (Imokawa et al, 1991). Cer- 
amides (non-hydroxy fatty acid and hydroxy fatty acid type; Sigma Chemical Company, St. Louis, MO) were used as standards for ceramides 1 and 2 and ceramides $3,4,5$, and 6 , respectively. The reproducibility of this method was confirmed using triplicate samples from the same subjects and the deviation of values (total lipids or micrograms of ceramide per milligram of stratum corneum) was within $5 \%$ of the means.

\section{Other methods}

The protein concentrations of tissue extracts were determined using a BCA protein assay kit (Pierce, Rockford, IL) with BSA as the standard.

\section{Statistical Analysis}

A nonparametric one-way ANOVA (Kruskal-Wallis test) was used to evaluate differences between groups. Where appropriate, a nonparametric post hoc multiple comparison test (Steel-Dwass test) was performed to evaluate differences between the groups. $P<0.05$ was considered statistically significant.

\section{References}

Akimoto K, Yoshikawa N, Higaki Y, Akimoto G, Yoshikawa N, Higaki Y, Kawashima M, and Imokawa G (1993). Quantitative analysis of stratum corneum lipids in xerosis and asteatotic eczema. J Dermatol 20:1-6.

Arikawa J, Ishibashi M, Kawashima M, Takagi Y, Ichikawa Y, and Imokawa G (2002). Decreased levels of sphingosine, a natural antimicrobial agent, may be associated with vulnerability of the stratum corneum from patients with atopic dermatitis to colonization by Staphylococcus aureus. J Invest Dermatol 119:433-439.

Atsumi S, Nosaka C, linuma H, and Umezawa K (1993). Accumulation of tissue glucosylsphingosine in Gaucher-like mouse induced by the glucosylceramidase inhibitor cyclophellitol. Arch Biochem Biophys 304:302-304.

Bouwstra JA, Dubbelaar FE, Gooris GS, Weerheim AM, and Ponec M (1999). The role of ceramide composition in the lipid organisation of the skin barrier. Biochim Biophys Acta 1419: 127-136.

Hanifin JM and Rajka G (1989). Diagnostic features of atopic dermatitis. Acta Derm Venereol (Stockh) 92:44-47.

Hara J, Higuchi K, Okamoto R, Kawashima M, and Imokawa G (2000). High-expression of sphingomyelin deacylase is an important determinant of ceramide deficiency leading to barrier disruption in atopic dermatitis. J Invest Dermatol 115:406-413.

Higuchi K, Hara J, Okamoto R, Kawashima M, and Imokawa $\mathrm{G}$ (2000). The skin of atopic dermatitis patients contains a novel enzyme, glucosylceramide sphingomyelin deacylase, which cleaves the $\mathrm{N}$-acyl linkage of sphingomyelin and glucosylceramide. Biochem J 350:747-756.

Imokawa G, Abe A, Jin K, Higaki Y, Kawashima M, and Hidano A (1991). Decreased level of ceramides in stratum corneum of atopic dermatitis: An etiologic factor in atopic dry skin? J Invest Dermatol 96:523-526.
Imokawa G, Yada Y, Higuchi K, Okuda M, Ohashi Y, and Kawamata A (1994). Pseudo-acylceramide with linoleic acid produces selective recovery of diminished cutaneous barrier function in essential fatty acid deficient rats and has an inhibitory effect on epidermal hyperplasia. J Clin Invest 94:89-96.

Ito M, Kurita T, and Kita K (1995). A novel enzyme that cleaves the $\mathrm{N}$-acyl linkage of ceramides in various glycosphingolipids as well as sphingomyelin to produce their lyso forms. J Biol Chem 270:24370-24374.

Jin K, Higaki Y, Takagi Y, Higuchi K, Yada Y, Kawashima M, and Imokawa G (1994). Analysis of $\beta$-glucocerebrosidase and ceramidase activities in atopic and aged dry skin. Acta Derm Venereol (Stockh) 74:337-340.

Kanazawa T, Nakamura S, Momoi M, Yamaji T, Takematsu H, Yano H, Sabe H, Yamanoto A, Kawasaki T, and Kozutsumi Y (2000). Inhibition of cytokinesis by a lipid metabolite, psychosine. J Cell Biol 149:943-950.

Murata Y, Ogata J, Higaki Y, Kawashima M, Yada Y, Higuchi K, Tsuchiya T, Kawaminami S, and Imokawa G (1996). Abnormal expression of sphingomyelin acylase in atopic dermatitis: An etiologic factor for ceramide deficiency? J Invest Dermatol 106:1242-1249.

Ohnishi Y, Okino N, Ito M, and Imayama S (1999). Ceramidase activity in bacterial skin flora as a possible cause of ceramide deficiency in atopic dermatitis. Clin Diagn Lab Immunol 6:101-104.

Okamoto R, Hara J, Kawashima M, Takagi Y, and Imokawa G (1999). Quantitative analysis of sphingosylphosphorylcholine in the stratum corneum of atopic dermatitis patients (Abstract). J Dermatol Sci 20:224.

Orbisky E, Sidransky E, McKinney CE, Lamarca ME, Samimi R, Krasnewich D, Martin BM, and Ginns El (2000). Glucosylsphingosine accumulation in mice and patients with type 2 Gaucher disease begins early in gestation. Pediatr Res 48:233-237.

Rawlings AV, Davies A, Carlomusto M, Pillai S, Zhang K, Kosturko R, Verdejo P, Feinberg C, Nguyen L, and Chandar $P$ (1996). Effect of lactic acid isomers on keratinocyte ceramide synthesis, stratum corneum lipid levels and stratum corneum barrier function. Arch Dermatol Res 288:383-390.

Schmuth M, Man MQ, Weber F, Gao W, Feingold KR, Fritsch P, Elias PM, and Holleran WM (2000). Permeability barrier disorder in Niemann-Pick disease: Sphingomyelin-ceramide processing required for normal barrier homeostasis. J Invest Dermatol 115:459-466.

Uchida Y, Hara M, Nishio H, Sidransky E, Inoue S, Otsuka F, Suzuki A, Elias PM, Holleran WM, and Hamanaka S (2000). Epidermal sphingomyelins are precursors for selected stratum corneum ceramides. J Lipid Res 41:2071-2082.

Umeda Y, Mizutani H, Imokawa G, and Shimizu M (1997). Topical ceramide corrected epidermal cell hyperproliferation and stratum corneum dysmaturation in atopic eczema. New Trends Allergy 4:237-239.

Yada Y, Higuchi K, and Imokawa G (1995). Purification and biochemical characterization of membrane-bound epidermal ceramidases from guinea pig skin. J Biol Chem 270:1267712684.

Yatomi Y, Ruan F, Ohta J, Welch RJ, Hakomori S, and Igarashi $Y$ (1995). Quantitative measurement of sphingosine 1-phosphate in biological samples by acylation with radioactive acetic anhydride. Anal Biochem 230:315-320. 